\title{
Los nuevos escenarios de la cooperación Sur-Sur entre Panamá y Marruecos
}

\section{S.E. Gloria Young ${ }^{1 *}$}

${ }^{1}$ Embajadora de Panamá en Marruecos

*Autor para correspondencia. Email:

Recibido: 09 de mayo de 2018

Aceptado: 03 de julio de 2018

\section{Resumen}

El presente ensayo forma parte fundamental de la investigación doctoral en curso, titulada: "Marruecos y la Cooperación Sur-Sur con Centroamérica y el Caribe", el mismo muestra los comienzos de ese espacio de entendimiento entre Panamá y Marruecos llamado Cooperación Sur - Sur; cómo a través de diálogos interculturales se manifiestan voluntades políticas que buscan develar experiencias contando historias. Siendo parte de un trabajo que transita por caminos no terminados, el presente ensayo más que concluir muestra como este Acuerdo sigue en el arduo trabajo de construir escenarios que buscan dar sentido a los significados hallados entre ambas naciones, traducidos en encuentros políticos, sociales y culturales.

\section{Construcción de nuevos escenarios: Inicios de un fenómeno llamado Cooperación Sur-} Sur.

El complejo mundo de las relaciones internacionales actuales, tras enfrentar los retos impuestos por la globalización, como la falta de una adecuada distribución de las riquezas, las exclusiones y el uso del poder militar como vía para resolver conflictos, ha conducido a un mundo marcado por nuevas relaciones de entendimiento, diálogo, respeto mutuo, consenso y cooperación, en todas las esferas de la vida.

La economía, la seguridad y la política, no son las únicas esferas donde la cooperación internacional, encuentra espacio y entendimiento. La comunidad global también está más dispuesta a convivir armonizando sus identidades y diferencias en pro de alcanzar caminos de paz, progreso y bienestar compartidos, de cara al futuro y a los retos que nos amenazan como naciones y como planeta.

Naciones como Marruecos y Panamá, no escapan a esa dinámica de trasformación diplomática a partir de historias comunes, problemas, retos y expectativas coincidentes. Ambos países comparten una mayor conciencia, traducida en acciones constantes, programadas en los diversos campos del quehacer humano y social. Se ha extendido en el escenario de la cooperación, el tapete de los recursos y ventajas, en áreas del comercio, la logística aérea, el turismo, la industria marítima, la educación, la cultura, la sostenibilidad del medio ambiente (Vagni, s/f). 
Hoy, se ha construido nuevos espacios en el mundo de la Cooperación Sur-Sur. Panamá y Marruecos han despertado ese destino histórico de grandes proporciones en la nueva diplomacia que el mundo contemporáneo necesita con decisión y voluntad. Un destino de urgentes transformaciones, pero ya no como meros espectadores, sino como protagonistas, actores de primer orden con propuestas afirmativas de presente y futuro, participando activamente en el desarrollo de la región, mediante una cooperación más humana, coordinada y eficiente.

Autores como Colacrai (2009), afirman que lo que se pretende es precisamente la construcción de "sociedades con mayor rasgo equitativo, "cohesionadas, en franco diálogo con el medio ambiente, con instituciones sólidas que garanticen una buena gobernabilidad, además de constituir metas a lograr por los estados en el marco de sus políticas nacionales, pueden estar potenciadas por la cooperación internacional" (p. 4).

Países como Marruecos, han visto la Cooperación Sur-Sur, como una estrategia, en una herramienta y en un componente fundamental de las políticas de desarrollo del país (Mohamed VI, 2016). El tema y las acciones van más allá de los resultados económicos medibles, ya que diseña y desarrolla proyectos en todos los ámbitos del desarrollo integral del país, incluyendo la parte religiosa.

La mirada transformada de Marruecos a América Latina hace que su liderazgo sea indiscutible en el tema de la Cooperación Sur - Sur; los proyectos con muchos países de África han sido su punta de lanza para lograr un apoyo histórico. La posibilidad de visualización y en el análisis exhaustivo de la relación de Marruecos con Centroamérica y el Caribe, específicamente con Panamá en el plano de la Cooperación Sur - Sur, es el objetivo principal de esta investigación. El desentrañar el papel que ha jugado Marruecos en la posible entrada de los países de Centroamérica y el Caribe, al escenario que presenta la Cooperación Sur - Sur, así como la existencia voluntad política regional para la entrada al juego propuesto, es la intención que recorre de manera transversal la investigación.

Es por lo anterior que la investigación que se lleva a cabo pretende la revisión exhaustiva de los hechos, recogidos en múltiples informes internacionales, agendas de gobiernos. Bajo una perspectiva cualitativa fundada en el paradigma de investigación postmoderno, de corte hermeneútico-histórico, el estudio busca precisamente dar cuenta del proceso, actores y escenarios donde se lleva a cabo la Cooperación Sur - Sur entre Marruecos y Panamá, apuntando tanto aciertos como limitaciones de este encuentro entre naciones. El método mencionado permite a partir de diálogos, acuerdos y alianzas, el encuentro con los protagonistas del fenómeno a estudiar. El discurso del sujeto deliberante, los actores gubernamentales (Panamá y Marruecos), girará en torno a la sociedad civil, al mundo privado, a la violencia de género, de la cultura, del deporte, de la vida orgánica de sus pueblos, el descubrimiento, la validación y la aplicación diferencian sus contextos y permitirá construir de manera clara el entramado de significados que envuelven la Cooperación Sur - Sur entre ambas naciones. 
Invest. pens. crit. (ISSN 1812-3864)

Vol. 6, No. 2, mayo-agosto 2018

pp. 85-92

\section{Todo tuvo una historia: Los sentidos en el encuentro entre naciones}

Las relaciones de América Latina y el mundo árabe, se remontan a un pasado de siglos a través de la España medieval y colonizadora, a partir del siglo XV, con lo que esto ha representado para América: las valiosas aportaciones del rico y vasto mundo musulmán; imán y semilla de las artes, la literatura, la filosofía y las ciencias. Aportes universales, que hoy incluso constituyen fuente para investigaciones en torno a ese encuentro maravilloso de dos mundos, en la construcción de ese puente, específico, entre Marruecos y Panamá, más que una coincidencia, es una búsqueda y un encuentro común impulsado por una política moderna y estratégica de entendimiento cultural, de progreso material para sociedades en ascenso, en lo que de común tienen y pueden ofrecer entre sí y al mundo.

Vagni (s/f), afirma que estos contactos, "que surgieron bajo el signo de la marginalidad y la discreción, atravesaron profundas oscilaciones y alcanzan hoy un elevado nivel de complejidad y densidad" (p. 2), sostiene que han puesto a prueba su capacidad para asumir nuevos desafíos, que implica dentro del escenario internacional una posición articulada. Se coincide con el autor, cuando comenta que al tratarse de regiones distantes en lo geográfico y, en cierta medida, también en lo cultural, el tratamiento de aquellos ámbitos que hacen al conocimiento mutuo entre ambos mundos se ha vuelto una cuestión esencial” (p. 2).

Después de una concurrencia diplomática desde Lisboa, por más de veinte años, un 18 de abril de 2014, se inauguró la embajada número 49 de Panamá, esta vez, en Marruecos ${ }^{1}$. Este paso, importante por demás, demarca el nuevo relacionar entre ambas naciones, el árbol de cooperación mutua dio sus frutos luego de atravesar un largo proceso de conversaciones y la puesta en marcha de acciones progresivas.

La expectativa inicial de esta apertura estuvo enmarcada por el interés de facilitar un comercio bilateral, el intercambio logístico aéreo y la consolidación del Acuerdo Marco: ACUERDO DE COOPERACION ENTRE EL GOBIERNO DE LA REPUBLICA DE PANAMA Y EL GOBIERNO DEL REINO DE MARRUECOS firmado en Rabat, el 17 de abril de 2014. Partiendo de esta tangible realidad, se desarrollaron otros acuerdos comerciales y logísticos en el campo aéreo.

Por otra parte, el Reino de Marruecos ya tiene embajada en Panamá desde el 18 de enero de 2016, Panamá por su parte cuenta con la embajada en Rabat y el Consulado de Marina Mercante en Tánger desde el 1 de noviembre de 2016, creado con el fin de potenciar las relaciones marítimas, portuarias y logísticas, entre los dos países.

Tal como lo dijo en su momento, el Administrador de la Autoridad Marítima de Panamá, Jorge Barakat ${ }^{2}$ :

Panamá es el país de referencia para acceder a los mercados de norte y sur América, y por su parte el reino de Marruecos, es un sitial clave

\footnotetext{
${ }^{1}$ Noticia reseñada en https://www.prensa.com/mundo/Panama-inaugura-Marruecos-embajadanumero_0_3915358441.html

${ }^{2}$ https://www.mire.gob.pa/index.php/en/noticias-mire/9942-
} 
Invest. pens. crit. (ISSN 1812-3864)

Vol. 6, No. 2, mayo-agosto 2018

pp. $85-92$

para el continente africano y europeo, por lo que ambos comparten

similitudes por ser puntos estratégicos comerciales, principalmente en cuanto a la navegación.

Panamá posee el registro de buques más grande del mundo, con aproximadamente ocho mil naves abanderadas y proporciona la solidez de la seguridad jurídica gubernamental, lo que le ha favorecido desde 1917. Además de ser el primer país en el mundo en implementar un sistema de registro de buques internacionales a nivel público, contando con la preferencia de importantes armadores y operadores de potencias marítimas internacionales, como China, Japón, Singapur y Corea del Sur, entre otros.

Tomando en cuenta lo anterior, es posible afirmar que Panamá representa un factor clave en la construcción de la Cooperación Sur - Sur, al poseer una experiencia vasta en áreas como las mencionadas. Este tipo de vínculos se hacen indispensables en un mundo necesitado de iniciativas productivas de cooperación e intercambio que potencien el desarrollo sostenible de naciones y regiones, sobre todo aquellas que han sido golpeadas por la pobreza y la negación al acceso a bienes y servicios públicos que ayudarían a elevar la calidad de vida de esas poblaciones, tanto de África, Asia como en América Latina, las regiones del Sur.

De este modo, Marruecos y Panamá ensayan un modelo de dinámica de cooperación internacional que busca perfeccionarse mediante acciones concretas en diversos campos, pero que además está abriendo puertas a nuevos escenarios de la Cooperación Sur-Sur, tales como la educación, las ciencias, la tecnología, y la cultura, entre otros intercambios. En estos nuevos escenarios, tienen cabida otros actores: los académicos, profesores universitarios, especialistas en diversas ramas del saber, técnicos, intelectuales, escritores, artistas, músicos, y otros miembros de la sociedad civil, como los jóvenes, las mujeres y grupos originarios, artesanos, ecologistas, entre otros.

\section{Los nuevos escenarios de la Cooperación Sur - Sur y la consolidación de oportunidades}

Los escenarios que dan lugar y apertura a la consolidación de la Cooperación Sur - Sur, se conciben para efectos del proyecto doctoral que realiza actualmente la autora, como espacios, lugares de acción, que rompen los modelos "tradicionales". No solo se quedan en los sectores productivos como comercio, navegación marítima, logística y turismo, sino que genera oportunidades en otros sectores del mundo académico y de la sociedad civil, gestando actividades de doble vía. Estas actividades representan acciones que a su vez inciden activamente entre ambas naciones, en las dinámicas internas de los países, a través de instancias democráticas y de participación, llenas de energía y entusiasmo.

Vagni (s/f), sostiene que la "diversificación de los vínculos entre América Latina y el mundo árabe-islámico ha puesto en evidencia la generación de nuevas instancias de acercamiento que pueden ser encuadradas en el marco de la diplomacia pública y cultural” (p. 3). El desarrollo de entramados ha sustentado este proceso complejo, de características cada vez más densas en aspectos políticos, sociales y culturales. Nuevos temas de la agenda, así como nuevos actores constituyen el rasgo más singular de esta aproximación 
Invest. pens. crit. (ISSN 1812-3864)

Vol. 6, No. 2, mayo-agosto 2018

pp. $85-92$

Son espacios con sentido de pertenencia, identidad y empoderamiento, que desarrollan ciencia y tecnología, educación y sostenibilidad ambiental, fomentando el rescate de la cultura originaria, la cultura en general. Espacios no muy ponderados por economistas y tecnócratas, pero que dan cuenta de mujeres y hombres comunes como sus principales beneficiarios.

Escenarios donde la innovación y la creatividad son la tónica, donde la investigación encuentra su sentido lógico traducido al servicio de elevar la calidad de vida de los ciudadanos y ciudadanas, donde la construcción de entornos más amables procuran el reverdecimiento de la vida, haciendo florecer ciudades, escuelas, y hasta pequeñas comunidades. Un ejemplo de lo comentado se evidencia en eventos realizados por diferentes Casas de estudio como aquellos llevados a cabo en la Universidad Ibn Zohr de Agadir ${ }^{3}$, donde la academia se une con el arte, e irrumpe en la cotidianidad de la sociedad marroquí, los estudiantes derrochan creatividad escénica en las calles y avenidas de la ciudad, llenando a los presentes de mundos fantásticos y reales magnificados; las emociones se concentran y destellan luces; la historia de las primeras civilizaciones es atrapada por el tiempo presente, para dejar las lecciones que aún deben aprenderse.

La cooperación internacional tradicional cubre ciertas expectativas, generando respuestas a necesidades y aspiraciones básicas de ciertos sectores de la sociedad y no de las grandes mayorías, que bien podrían necesitar carreteras, puentes peatonales, hospitales, trabajo, canchas deportivas. Sí, bien es ciert lo expuesto, también es cierto que la Cooperación Sur Sur también se dedica a esos espacios necesarios y a otros, como los que se han mencionado a lo largo del documento.

Sin embargo, es importante señalar que los nuevos escenarios de la Cooperación Sur Sur contribuyen al conocimiento de los pueblos, sus culturas, artes, tradiciones y costumbres, las cuales contribuyen a fortalecer las idiosincrasias y "haceres", es decir a aquello que se ha sido en el tiempo; eso que se es y se quiere ser, el sentido social de la existencia de los pueblos, y la relación armoniosa con otros y sus culturas.

Desde la cooperación específica a la que se refiere en este ensayo, se contempla la noción que todo lo que ayude al conocimiento pleno propio y al crecimiento como humanos, como seres comunicativos, pacíficos y solidarios, respetuosos de la vida y del entorno, generará acciones que fomentará valores trascedentes que atraviesen fronteras y aproximen a los pueblos a metas comunes, contemplando la de exploración de mejores alternativas de solución a los problemas más esenciales del ser humano y de la sociedad en general.

En el accionar de estos escenarios se busca de forma activa la construcción de un mundo mejor, promoviendo la consolidación de democracias sólidas cimentadas en la igualdad, la equidad, la libertad, la justicia, y el respeto de todos los derechos humanos. En el caso de Marruecos y Panamá, se ha puesto en marcha acciones en estos nuevos espacios, que permitan abrir nuevos caminos de cooperación social y humana, en este mundo Sur-Sur más solidario, que se intenta levantar frente a la bipolaridad disfuncional de un norte siempre rico y un sur siempre pobre. Con estas acciones, a partir de los mismos protagonistas, ser un espejo para las otras naciones de la región, África y América Latina.

\footnotetext{
${ }^{3}$ http://www.uiz.ac.ma/
} 
De los múltiples ejemplos del accionar sobre estos escenarios, se cita el proyecto entre la Universidad de Panamá, coordinado en su Centro Regional Universitario de Coclé, provincia ubicada en el corazón del territorio panameño y el Laboratorio de Investigación del mundo ibero e iberoamericano de la Universidad Hassan II de Casablanca. Se trató de una investigación de la historia de Marruecos y de Panamá desde la voz poética de sus mujeres; poetas, narradoras de historias de ambos países de la primera y última generación. Una línea de investigación que promocionó a ambas naciones, en base a la riqueza de su diversidad cultural, visibilizando la historia de sus pueblos desde la construcción de las identidades hasta las manifestaciones más contemporáneas.

Se entabló un diálogo intercultural que posibilitó el redescubrimiento mutuo; un intercambio que permitiera conocer y cimentar puntos en común desde realidades diversas. Este diálogo de culturas vivo se posibilitó, sobre todo, por mujeres investigadoras y hombres solidarios. Una gama de actores, construyendo nuevas habilidades, así como actitudes de apertura, confianza, respeto y aprendizaje mutuo. Una Cooperación Sur - Sur que utilizó la historia y la literatura como una herramienta para el desarrollo desde la diplomacia cultural en este mundo mucho más complejo.

Se rescataron de los cajones polvorientos de la crítica literaria tendenciosa y del estudio de la historia que oculta a los verdaderos protagonistas, la voz de las mujeres. El libro, escrito en árabe y español, producto de esta investigación, se ha presentado en Marruecos y Panamá, en más veinte ocasiones a otros actores, en otros escenarios de la Cooperación Sur - Sur. Los logros que dan cuenta de lo planteado es la ausencia de este libro en los estantes olvidados de alguna biblioteca, o en los libreros personales de los investigadores y unos cuantos lectores. Es un libro vivo que propone una metodología del estudio de la historia, desde la creación literaria contextual, permitiendo la exposición de la producción de mujeres poetas olvidadas en la historia, que la mayor parte de las veces, escriben los hombres. Así, ambos pueblos conocen su historia y la del otro.

Otro proyecto innovador, aún en curso, es el de los "Diálogos entre bordados y tejidos originarios de Panamá y Marruecos". Desde la Cooperación Sur - Sur en escenarios inimaginables, dos pueblos se acercan de manera creativa, potenciando la cultura y el ecoturismo. Artesanas de los pueblos originarios gunas y wounan, disfrutan una estancia cultural compartiendo habilidades con artesanas marroquíes, estudiantes de bordados tradicionales de este territorio Alahui; facilitando talleres con mujeres de diversas culturas y grados académicos, compartiendo con artesanos de la famosa ciudad azul de Chefchauen y trabajando en el taller de una de las diseñadoras de moda más prestigiosas de mundo árabe, proveniente de la cultura hasaní, Fadila El Gadi. Es un proyecto que llevó a esta diseñadora a adentrarse a las montañas de la comarca Nagbe Buglé, a penetrar la selva del Darién, frontera con Colombia, y a transitar en las aguas rugientes del caribe panameño para alcanzar islas de la comarca Guna Yala.

Un proyecto, que traspasa la visión tradicional de los ministerios de turismo y casas de artesanía, cambiando la usual feria artesanal y por teatros expuestos vestidos de arte, cultura e historia. La pasarela intercultural como fase final del proyecto da cuenta de la esencia central de este maravilloso escenario colorido y asombroso, el diálogo propuesto por dos diseñadores de países 
Invest. pens. crit. (ISSN 1812-3864)

Vol. 6, No. 2, mayo-agosto 2018

pp. $85-92$

tan diversos, gira en torno a sus raíces, la afrodescendiente de la provincia caribeña de Colón, y la africana, con toques saharaui.

Otro espacio que habla de las experiencias inmersas en la Cooperación Sur - Sur, es el académico, donde Panamá inaugura la primera Maestría: "América Latina: globalización, interculturalidad y desafíos en el siglo XXI", de la Universidad Mohammed V. En ese escenario, cerca de treinta estudiantes, se abren al estudio profundo de región centroamericana, en sus contexto histórico, social, económico y político. De la mano de un estudioso panameño, los participantes podrán adentrarse a la historia de Centroamérica profunda, para generar experiencias desde perspectivas interculturales, con diálogos conscientes, reconociendo al otro plural, diverso y diferente.

\section{E1 tiempo de Agadir: Conclusiones de un proyecto no acabado}

Nunca como antes en la historia de las relaciones entre Panamá y Marruecos, con plena conciencia de ser parte y protagonistas de sus historias, y de compartir metas comunes de desarrollo y cooperación, se han generado tantos acuerdos, convenios y acciones que apuntan al trabajo armónico de ambas naciones, y el compromiso de ofrecer lo mejor de cada una para el crecimiento mutuo. El compartir expreso con países hermanos de la región, se evidencia en los frutos recogidos de esta nueva experiencia de Cooperación Sur - Sur reafirmando que se recorre el camino correcto, por el bien de los pueblos, la región que se habita, y el mundo que se pretende y quiere construir con granos de arena y olas de mar.

Los escenarios compartidos hasta ahora desde la Cooperación Sur - Sur son múltiples, diversos, interculturales y mágicos. El convencimiento de que los esfuerzos hechos desde la diplomacia con una visión humana y de futuro, es el trabajo diario sin descanso, para el cumplimiento eficaz, responsable, ético de todas las metas propuestas que acerquen de manera indiscutible a los objetivos que las naciones reunidas en torno a la ONU se han comprometido a realizar, con liderazgo, creatividad y sentido humano.

Los nuevos escenarios de la Cooperación Sur - Sur no tienen límites. Depende del nivel de compromiso y de las capacidades de los protagonistas involucrados en la labor de sumar actores a la causa: una nueva relación de poder, un nuevo pacto con trato igualitario y respetuoso entre las naciones, los pueblos y los seres humanos. En este sentido se afirma a partir de lo expuesto: No existe la posibilidad de callar, ni ser imparciales. Tomando como ciertas las palabras del científico inglés Hawking (1942-2018), dijo: "El peligro radica en que nuestro poder para dañar o destruir el medio ambiente, o al prójimo, aumenta a mucha mayor velocidad que nuestra sabiduría en el uso de ese poder".

La invitación que se hace a partir de las reflexiones presentadas y del trabajo en curso es el uso sincero del poder, de las energías para la cooperación pretendida entre todos en la construcción de un mundo mejor, del antes soñado y ahora posible. El mundo que Marruecos y Panamá han decidido asumir, desde las identidades compartidas; en la construcción hermanada de ese feliz destino que los espera a la vuelta de la esquina. 
Invest. pens. crit. (ISSN 1812-3864)

Vol. 6, No. 2, mayo-agosto 2018

pp. $85-92$

\section{Bibliografía}

Colacrai, M., Kern, A. (2009). Escenarios y Desafios de la Cooperación Sur Sur a 30 años de la Declaración de Buenos Aires. Buenos Aires: Universidad Nacional de San Martín.

Klenger, Susanne; Ortiz Wallner, Alexandra (2016). Sur South - Poetics and Politics of thinking Latin America/India. Biblioteca Ibero-Americana 163 ; Madrid, España.

Mohamed VI (2016). Discurso real dirigido a la decimotercera cumbre de la Conferencia islámica de Estambul.

Vagni, J. (s/F). América Latina y el mundo árabe: un acercamiento desde la diplomacia pública y la cooperación cultural. Disponible:

http://web.isanet.org/Web/Conferences/FLACSOISA \%20BuenosAires \%202014/Archive/c58a6c98-e430-4857-8bde-e43b5ca7a57e.pdf

Panamá inaugura en Marruecos su embajada número 49. (18 de abril de 2014). La Prensa, p. 10

Panamá Oficializa la Apertura de su Consulado en el Reino de Marruecos. 08 November 2016. Ministerio de Relaciones Exteriores. Panamá. Disponible:

http://www.uiz.ac.ma/ 